\title{
MOA-2010-BLG-073L: AN M-DWARF WITH A SUBSTELLAR COMPANION AT THE PLANET/BROWN DWARF BOUNDARY
}

\author{
R. A. Street ${ }^{1}$, J.-Y. Chol $^{2}$, Y. Tsapras ${ }^{1,3}$, C. Han $^{2,65}$, K. Furusama ${ }^{4}$, M. Hundertmark ${ }^{5}$, A. Gould ${ }^{6}$, T. Sumi ${ }^{7}$, I. A. Bond ${ }^{8}$, \\ D. Wouters ${ }^{9}$, R. Zellem ${ }^{10}$, A. UdAlski ${ }^{11}$
}

AND

(The Robonet Collaboration)

C. Snodgrass ${ }^{12}$, K. Horne ${ }^{5}$, M. DominiK ${ }^{5,66}$, P. Browne ${ }^{5}$, N. Kains ${ }^{13}$, D. M. Bramich ${ }^{13}$, D. BajeK ${ }^{5}$, I. A. Steele ${ }^{14}$, S. Ipatov $^{15}$ AND

(The MOA Collaboration)

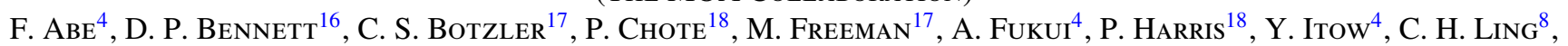
K. Masuda ${ }^{4}$, Y. Matsubara ${ }^{4}$, N. MiyaKe ${ }^{4}$, Y. Muraki ${ }^{19}$, T. NAgayama ${ }^{20}$, S. Nishimaya $^{21}$, K. Ohnishi $^{22}$, N. Rattenbury ${ }^{17}$, To. Saito $^{23}$, D. J. Sullivan ${ }^{18}$, D. Suzuki ${ }^{7}$, W. L. Sweatman ${ }^{8}$, P. J. Tristram ${ }^{24}$, K. Wada ${ }^{7}$, P. C. M. Yock ${ }^{17}$

AND

(The OGLE COLlaboration)

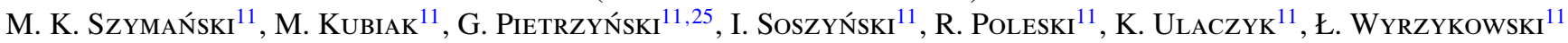

AND

(THE $\mu$ FUN COLlaboration)

J. YeE ${ }^{6}$, S. Dong ${ }^{26}$, I.-G. Shin ${ }^{2}$, C.-U. LeE ${ }^{27}$, J. Skowron ${ }^{6}$, L. Andrade De Almeida ${ }^{28}$, D. L. DePoy ${ }^{29}$, B. S. Gaudi ${ }^{6}$, L.-W. Hung ${ }^{6}$, F. JAblonski ${ }^{28}$, S. KASPI ${ }^{30}$, N. KLein ${ }^{30}$, K.-H. Hwang ${ }^{2}$, J.-R. $\mathrm{KoO}^{27}$, D. MAOz ${ }^{30}$, J. A. MuÑoz ${ }^{31}$, R. W. PogGE ${ }^{6}$, D. Polishrook ${ }^{30}$, A. ShPorer ${ }^{1,29}$, J. McCormick ${ }^{32}$, G. Christie ${ }^{33}$, T. Natusch ${ }^{33}$, B. Allen ${ }^{34}$, J. Drummond ${ }^{35}$,

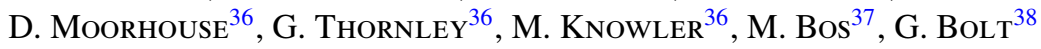

AND

(The Planet Collaboration)

J.-P. Beaulieu ${ }^{9}$, M. D. Albrow ${ }^{39}$, V. Batista ${ }^{6}$, S. Brillant ${ }^{40}$, J. A. R. CAlddwell ${ }^{41}$, A. Cassan ${ }^{42}$, A. Cole ${ }^{43}$, E. Corrales ${ }^{42}$, Ch. Coutures ${ }^{42}$, S. Dieters ${ }^{43}$, D. Dominis Prester ${ }^{44}$, J. Donatowicz ${ }^{45}$, P. Fouqué ${ }^{46,47}$, E. Bachelet ${ }^{46,47}$, J. Greenhill ${ }^{43}$, S. R. Kane ${ }^{48}$, D. $\mathrm{Kubas}^{47}$, J.-B. Marquette ${ }^{42}$, R. Martin ${ }^{49}$, J. Menzies ${ }^{50}$, K. R. Pollard ${ }^{17}$, K. C. Sahu ${ }^{51}$, J. WambsGanss ${ }^{52}$, A. Williams ${ }^{49}$, M. ZuB ${ }^{52}$

AND

(MINDSTEP)

K. A. Alsubai ${ }^{15}$, V. BozzA ${ }^{53}$, M. J. Burgdorf ${ }^{54}$, S. CAlchi Novati ${ }^{55}$, P. Dodds ${ }^{5}$, S. Dreizler ${ }^{56}$, F. Finet $^{57}$, T. Gerner $^{52}$,

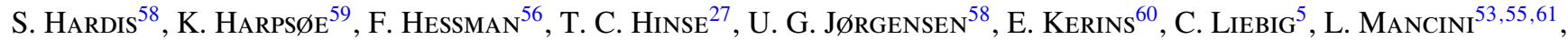

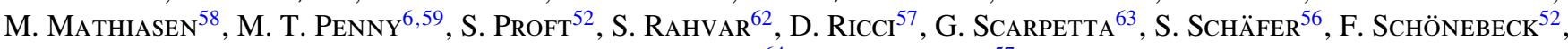
J. SOUTHWORTH ${ }^{64}$, AND J. SURDEJ ${ }^{57}$

${ }^{1}$ LCOGT, 6740 Cortona Drive, Suite 102, Goleta, CA 93117, USA; rstreet@1cogt.net

${ }^{2}$ Department of Physics, Institute for Astrophysics, Chungbuk National University, Cheongju 361-763, Republic of Korea

${ }^{3}$ School of Mathematical Sciences, Queen Mary, University of London, Mile End Road, London E1 4NS, UK

${ }^{4}$ Solar-Terrestrial Environment Laboratory, Nagoya University, Nagoya 464-8601, Japan

${ }^{5}$ SUPA/St Andrews, Department of Physics and Astronomy, North Haugh, St. Andrews, Fife KY16 9SS, UK

${ }^{6}$ Department of Astronomy, Ohio State University, McPherson Laboratory, 140 West 18th Avenue, Columbus, OH 43210-1173, USA

${ }^{7}$ Department of Earth and Space Science, Graduate School of Science, Osaka University, 1-1 Machikaneyama-cho, Toyonaka, Osaka 560-0043, Japan

${ }^{8}$ Institute of Information and Mathematical Sciences, Massey University, Private Bag 102-904, North Shore Mail Centre, Auckland, New Zealand

${ }^{9}$ UPMC-CNRS, UMR 7095, Institut d'Astrophysique de Paris, 98bis boulevard Arago, F-75014 Paris, France

${ }^{10}$ Lunar and Planetary Laboratory, Department of Planetary Sciences, University of Arizona, 1629 East University Boulevard, Tucson, AZ 85721-0092, USA

${ }^{11}$ Warsaw University Observatory, Al. Ujazdowskie 4, 00-478 Warszawa, Poland

${ }^{12}$ Max Planck Institute for Solar System Research, Max-Planck-Str. 2, D-37191 Katlenburg-Lindau, Germany

${ }^{13}$ European Southern Observatory, Karl-Schwarzschild-Straße 2, D-85748 Garching bei München, Germany

${ }^{14}$ Astrophysics Research Institute, Liverpool John Moores University, Twelve Quays House, Egerton Wharf, Birkenhead, Wirral CH41 1LD, UK ${ }^{15}$ Qatar Foundation, P.O. Box 5825, Doha, Qatar

${ }^{16}$ Department of Physics, University of Notre Dame, Notre Dame, IN 46556, USA

${ }^{17}$ Department of Physics, University of Auckland, Private Bag 92019, Auckland, New Zealand

${ }^{18}$ School of Chemical and Physical Sciences, Victoria University, Wellington, New Zealand

${ }^{19}$ Department of Physics, Konan University, Nishiokamoto 8-9-1, Kobe 658-8501, Japan

${ }^{20}$ Department of Physics and Astrophysics, Faculty of Science, Nagoya University, Nagoya 464-8602, Japan

${ }^{21}$ Extrasolar Planet Detection Project Office, National Astronomical Observatory of Japan (NAOJ), Osawa 2-12-1, Mitaka, Tokyo 181-8588, Japan ${ }^{22}$ Nagano National College of Technology, Nagano 381-8550, Japan

${ }^{23}$ Tokyo Metropolitan College of Industrial Technology, Tokyo 116-8523, Japan

${ }^{24}$ Mt. John Observatory, P.O. Box 56, Lake Tekapo 8770, New Zealand

${ }^{25}$ Universidad de Concepción, Departamento de Astronomia, Casilla 160-C, Concepción, Chile

${ }^{26}$ Institute for Advanced Study, Einstein Drive, Princeton, NJ 08540, USA

${ }^{27}$ Korea Astronomy \& Space Science Institute, Daejeon 305-348, Republic of Korea

${ }^{28}$ Divisao de Astrofisica, Instituto Nacional de Pesquisas Espeaciais, Avenida dos Astronauntas, 1758 Sao José dos Campos, $12227-010$ SP, Brazil

${ }^{29}$ Department of Physics and Astronomy, Texas A\&M University College Station, TX 77843-4242, USA

${ }^{30}$ School of Physics and Astronomy and Wise Observatory, Tel-Aviv University, Tel-Aviv 69978, Israel 


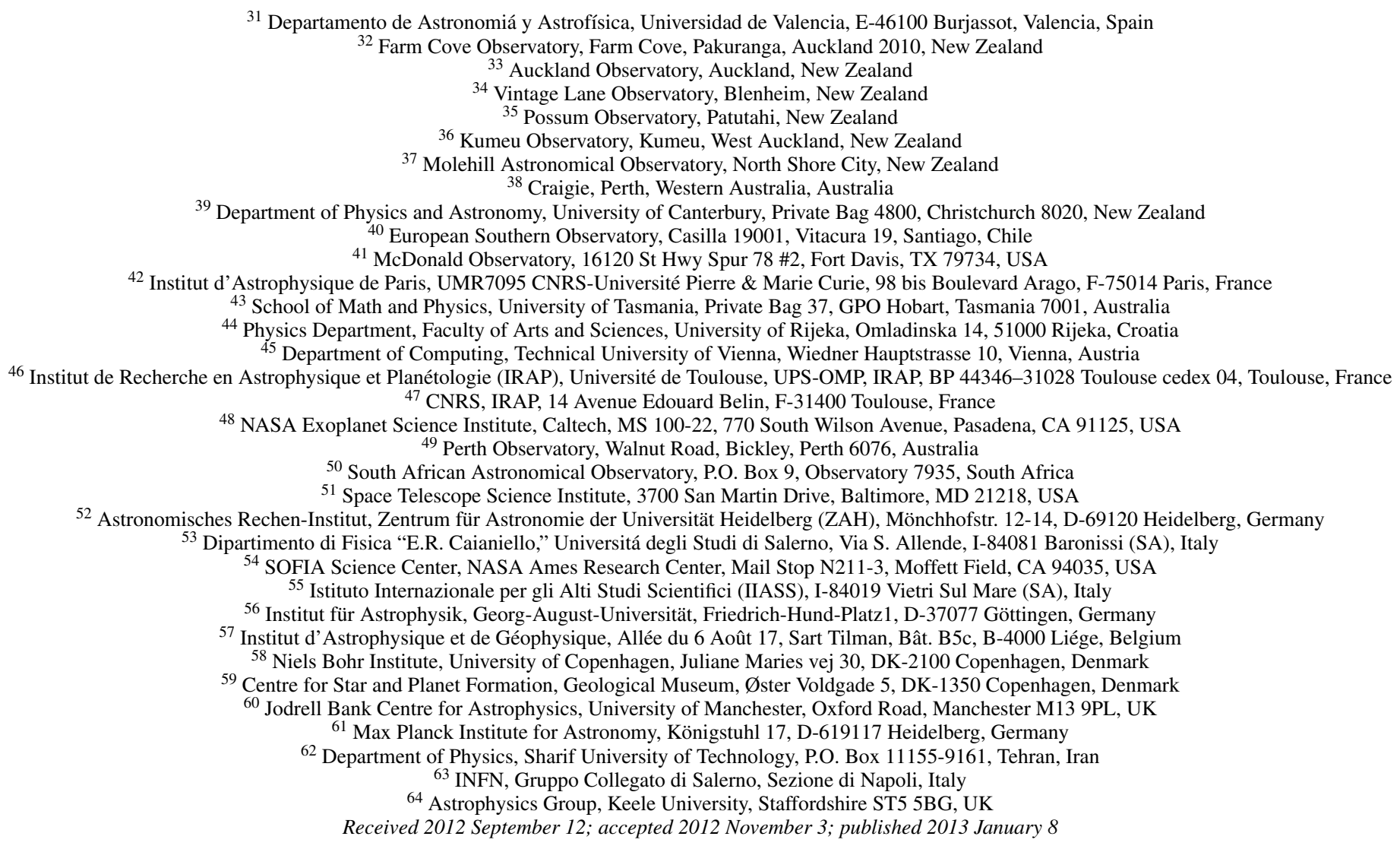

\section{ABSTRACT}

We present an analysis of the anomalous microlensing event, MOA-2010-BLG-073, announced by the Microlensing Observations in Astrophysics survey on 2010 March 18. This event was remarkable because the source was previously known to be photometrically variable. Analyzing the pre-event source light curve, we demonstrate that it is an irregular variable over timescales $>200$ days. Its dereddened color, $(V-I)_{S, 0}$, is $1.221 \pm 0.051 \mathrm{mag}$, and from our lens model we derive a source radius of $14.7 \pm 1.3 R_{\odot}$, suggesting that it is a red giant star. We initially explored a number of purely microlensing models for the event but found a residual gradient in the data taken prior to and after the event. This is likely to be due to the variability of the source rather than part of the lensing event, so we incorporated a slope parameter in our model in order to derive the true parameters of the lensing system. We find that the lensing system has a mass ratio of $q=0.0654 \pm 0.0006$. The Einstein crossing time of the event, $t_{\mathrm{E}}=$ $44.3 \pm 0.1$ days, was sufficiently long that the light curve exhibited parallax effects. In addition, the source trajectory relative to the large caustic structure allowed the orbital motion of the lens system to be detected. Combining the parallax with the Einstein radius, we were able to derive the distance to the lens, $D_{L}=2.8 \pm 0.4 \mathrm{kpc}$, and the masses of the lensing objects. The primary of the lens is an M-dwarf with $M_{L, 1}=0.16 \pm 0.03 M_{\odot}$, while the companion has $M_{L, 2}=11.0 \pm 2.0 M_{\mathrm{J}}$, putting it in the boundary zone between planets and brown dwarfs.

Key words: brown dwarfs - gravitational lensing: micro - planetary systems - planets and satellites: formation - planets and satellites: general - techniques: photometric

Online-only material: color figures

\section{INTRODUCTION}

The mass function of individual compact objects (brown dwarfs and planets) in the Galaxy remains poorly understood, particularly at the low-mass end. Brown dwarfs are commonly defined as objects with masses between the deuterium- and hydrogen-burning limits (DBL and HBL, respectively), but these can be hard to detect, being intrinsically faint and fading further as they cool over time. Below the DBL, the mass

\footnotetext{
65 Corresponding author.

${ }^{66}$ Royal Society University Research Fellow.
}

function for individual objects is even more poorly measured. Unbound, free-floating objects of planetary mass have been discovered via direct imaging of clusters (for example, in $\sigma$ Orionis; Béjar et al. 2012) and in the field (e.g., the 6-25 $M_{\mathrm{J}}$ object reported by Kirkpatrick et al. 2006). Sumi et al. (2011) reported a population of planets that are either unbound or at very wide separations, discovered when their gravity caused short-timescale microlensing events.

At least partially as a result of these poor constraints, the origin of low-mass compact objects remains unclear. Although traditionally thought of as separate classes of objects, planets and brown dwarfs form a continuous scale of mass and are 
best distinguished by the circumstances of their formation (Burrows et al. 2001; Chabrier et al. 2005, 2011; Sahlmann et al. 2010). Planets form in disks of material orbiting a protostellar object and may subsequently migrate to different period orbits. Brown dwarfs, on the other hand, are considered to be the extreme low-mass end of the star formation process by fragmentation of locally overdense cores caused by turbulence in a cloud (Chabrier et al. 2011), which can themselves form protoplanetary disks (Klein et al. 2003; Scholz et al. 2006). This mechanism may also produce objects of a few Jupiter masses. For a recent review, see Luhman (2012).

The mass function of free-floating low-mass objects is likely to be different from those bound to stars. Marcy \& Butler (2000) identified a paucity of brown dwarfs orbiting close $(<3 \mathrm{AU})$ to their host stars, a region where Jovian-mass planets are commonly found. This "brown dwarf desert" may represent the gap between the largest objects that can form in protoplanetary disks and the smallest objects that can concurrently collapse/ condense next to a star.

Two different theories have been proposed to explain the formation of Jovian planets in disks (for a review, see Zhou et al. 2012). The core accretion model predicts that planets form from protoplanetary cores, growing up to tens of Jupiter masses (e.g., Mordasini et al. 2009; Baraffe et al. 2010), but predicts few giant planets around M-dwarfs. Higher mass stars are thought to have disks with enhanced surface densities that allow the cores to grow more rapidly (Laughlin et al. 2004), as do disks with a high fraction of dust, leading to enhanced planet formation around high-metallicity stars (Ida \& Lin 2004). Alternatively, the model of planet formation via gravitational instabilities in the disk (e.g., Boss 2006) tends to favor the formation of more massive planets, in generally wider orbits.

A number of lines of evidence support the core accretion theory. There is a well-established correlation of increasing planet frequency with stellar metallicity (Santos et al. 2001; Fischer \& Valenti 2005; Maldonado et al. 2012). The results of radial velocity surveys imply that there is a dearth of M-dwarf stars with massive, close-in planets. In part, this reflects an observational bias against these faint objects, but the sample is sufficiently large that a real statistical trend is emerging (Cumming et al. 2008; Johnson et al. 2010; Bonfils et al. 2011), for companions with $P<2000$ days. Recent spectroscopic and Kepler results have confirmed the prediction of a rapid increase in frequency for planets with small radii (down to $2 R_{\oplus}$ ) and $P<50$ days for all spectral types and found that these small planets are several times more common around stars of late spectral type (Bonfils et al. 2011; Howard et al. 2012).

However, the core accretion model has difficulty forming massive planets at large orbital radii, and such systems have been discovered, for example, HR 8799 (Marois et al. 2008). Furthermore, a number of planets have been found orbiting M-dwarf hosts at larger orbital separations, for example, Dong et al. (2006), Forveille et al. (2011), and Batista et al. (2011). These systems may instead form through gravitational instability in the disk, which can account for companions up to several Jupiter masses around M-dwarfs (Boss 2006).

To better understand the formation mechanisms of heavy substellar companions in bound systems, we need to trace the distributions of the physical and orbital properties (such as mass ratio, orbital separation, occurrence frequency) of a significant number of systems. Yet relatively few bound brown dwarf companions have been reported, despite their being easy to detect at close orbital separations (the "brown dwarf desert").
Microlensing offers a complementary window onto brown dwarf and planet formation by probing for cooler companions of all masses in orbital radii between $\sim 0.2$ and $10 \mathrm{AU}$, separations that are difficult or time-consuming to explore by other methods (Shin et al. 2012b). It can probe the companion mass function down to $\mathrm{M}$ - and brown dwarf hosts and is sensitive to companions from nearly equal mass down to terrestrial masses.

Sixteen systems have been published to date, ${ }^{67}$ and thanks to large-scale galactic lensing surveys and efficient follow-up, each season's bulge observing campaign is now producing a regular yield of new discoveries (e.g., Bachelet et al. 2012; Yee et al. 2012; Miyake et al. 2012). Of these 16 companions, 3 are giant planets orbiting M-dwarf stars: OGLE-2005-BLG-071Lb, a 3.8 $M_{\text {J }}$ planet (Dong et al. 2009); MOA-2009-BLG-0387Lb, with an $M_{P}=2.5 M_{\mathrm{J}}$ planet (Batista et al. 2011); and MOA2011-BLG-293Lb, which hosts a $2.4 M_{\mathrm{J}}$ companion (Yee et al. 2012).

Here we present the newly discovered system, MOA-2010BLG-073L, an M-dwarf star with a companion whose mass is close to the DBL of $\sim 12.6 M_{\mathrm{J}}$. The discovery and followup observations are described in Section 2, and we discuss the variability of the source star in Section 3. We describe our analysis in Section 4, from which we derive the physical properties of the lens in Section 5. Finally, we discuss our findings in Section 6.

\section{OBSERVATIONS}

The microlensing event, MOA-2010-BLG-073, was first announced by the Microlensing Observations in Astrophysics ${ }^{68}$ (MOA; Bond et al. 2001 and Sumi et al. 2003 on the $1.8 \mathrm{~m}$ telescope at Mt. John Observatory, New Zealand) survey on 2010 March 18. A background source star in the Galactic bulge, $\alpha=18: 10: 11.342, \delta=-26: 31: 22.544$ (J2000.0), previously having a mean baseline magnitude of $I \sim 16.5 \mathrm{mag}$, was discovered to be rising smoothly in brightness consistent with a point-source, point-lens (PSPL) microlensing event. However, on 2010 May 3 the event was found to show an anomalous brightening of $\sim 0.5 \mathrm{mag}$ and an alert was issued (K. Furusawa 2010 , private communication).

Microlensing follow-up teams worldwide-RoboNet-II ${ }^{69}$ (Tsapras et al. 2009), $\mu \mathrm{FUN}^{70}$ (Gould et al. 2006), PLANET $^{71}$ (Beaulieu et al. 2006), and MiNDSTEp ${ }^{72}$ (Dominik et al. 2010)—responded to provide intensive coverage of the event for the duration of the anomaly ( $\sim 2$ days) and monitored the event as it returned to baseline, over the course of the next $\sim 2$ months.

In addition to the MOA data, taken with the wide-band "MOA-Red" filter (corresponding to $R+I$ bandpasses), the event was observed from several other sites in New Zealand. The $0.41 \mathrm{~m}$ telescope at Auckland Observatory, the $0.35 \mathrm{~m}$ at Kumeu Observatory, and the $0.41 \mathrm{~m}$ Possum Observatory all used $R$-band filters while the $0.304 \mathrm{~m}$ Molehill Astronomical Observatory (MAO), the $0.35 \mathrm{~m}$ telescope at Farm Cove Observatory (FCO), and the $0.4 \mathrm{~m}$ telescope at Vintage Lane Observatory (VLO) all observed it unfiltered. The event was then picked up

\footnotetext{
67 Listed on www.exoplanet.eu

68 www.phys.canterbury.ac.nz/moa

69 robonet.lcogt.net

70 www.astronomy.ohio-state.edu/ microfun

71 www.planet.iap.fr

72 www.mindstep-science.org
} 
Table 1

Summary of Observations

\begin{tabular}{|c|c|c|c|c|c|c|c|}
\hline $\begin{array}{l}\text { Telescope and } \\
\text { Aperture (m) }\end{array}$ & Filter & $u_{\lambda}{ }^{\mathrm{a}}$ & $\Gamma_{\lambda}^{a}$ & $\begin{array}{c}N \text { Frames } \\
\text { Total }\end{array}$ & $\begin{array}{c}N \text { Frames } \\
\text { Used }\end{array}$ & $a_{0}{ }^{\mathrm{a}}$ & $\begin{array}{c}a_{1}^{\mathrm{a}} \\
(\mathrm{mag})\end{array}$ \\
\hline MOA 1.8 & $R / I$ & 0.7027 & 0.6118 & 1747 & 1726 & 1.305 & 0.005 \\
\hline OGLE $1.3^{\mathrm{b}}$ & $I$ & 0.6098 & 0.5103 & 47 & 42 & 2.188 & 0.005 \\
\hline Auckland 0.41 & $R$ & 0.7027 & 0.6118 & 136 & 136 & 0.910 & 0.000 \\
\hline Canopus 1.0 & $I$ & 0.6098 & 0.5103 & 162 & 159 & 1.310 & 0.005 \\
\hline CTIO 1.3 & $V$ & 0.7817 & 0.7048 & 19 & 18 & 0.603 & 0.000 \\
\hline CTIO 1.3 & $I$ & 0.6098 & 0.5103 & 162 & 162 & 1.010 & 0.003 \\
\hline CTIO 1.3 & $H$ & 0.4145 & 0.3206 & 586 & 575 & 1.340 & 0.016 \\
\hline Danish 1.54 & $I$ & 0.6098 & 0.5103 & 498 & 491 & 1.130 & 0.014 \\
\hline Farm Cove $0.4^{\mathrm{c}}$ & Unfiltered & $\ldots$ & 0.5611 & 225 & 225 & 0.975 & 0.000 \\
\hline FTN 2.0 & SDSS- $i$ & 0.6098 & 0.5103 & 159 & 158 & 1.055 & 0.011 \\
\hline FTS 2.0 & SDSS- $i$ & 0.6098 & 0.5103 & 129 & 129 & 1.125 & 0.006 \\
\hline IRSF 1.4 & $J$ & 0.4836 & 0.3844 & 4 & 4 & 1.000 & 0.000 \\
\hline IRSF 1.4 & $H$ & 0.4145 & 0.3206 & 4 & 4 & 1.000 & 0.000 \\
\hline IRSF 1.4 & $K_{S}$ & 0.3550 & 0.2684 & 4 & 4 & 1.000 & 0.000 \\
\hline Kumeu 0.35 & $R$ & 0.7027 & 0.6118 & 272 & 272 & 0.772 & 0.000 \\
\hline Lemmon 1.0 & $I$ & 0.6098 & 0.5103 & 116 & 105 & 1.290 & 0.020 \\
\hline LT 2.0 & SDSS- $i$ & 0.6098 & 0.5103 & 167 & 167 & 1.155 & 0.006 \\
\hline $\mathrm{MAO} 0.304^{\mathrm{c}}$ & Unfiltered & $\ldots$ & 0.5611 & 238 & 238 & 1.025 & 0.018 \\
\hline Perth 0.6 & $I$ & 0.6098 & 0.5103 & 66 & 66 & 1.095 & 0.008 \\
\hline Possum & $R$ & 0.7027 & 0.6118 & 15 & 15 & 1.030 & 0.009 \\
\hline SAAO 1.0 & $I$ & 0.6098 & 0.5103 & 30 & 30 & 1.050 & 0.011 \\
\hline Vintage Lane $0.4^{\mathrm{c}}$ & Unfiltered & $\ldots$ & 0.5611 & 124 & 124 & 0.995 & 0.000 \\
\hline
\end{tabular}

Notes.

${ }^{a} u_{\lambda}, \Gamma_{\lambda}$ are defined by Equations (3), (4), (5) and $a_{0}, a_{1}$ by Equation (6).

${ }^{b}$ Includes only OGLE-IV data taken during event MOA-2010-BLG-073.

${ }^{\mathrm{c}}$ For unfiltered or very broadband observations we adopted a limb-darkening parameter value that was the average of that for $R$ and $I$ bands: $\left(\Gamma_{R}+\Gamma_{I}\right) / 2$.

from three sites in Australia, first in $I$ band by the $1 \mathrm{~m}$ Canopus telescope in Tasmania followed by the $2 \mathrm{~m}$ Faulkes Telescope South (FTS), where an SDSS- $i$ filter was used. The $0.6 \mathrm{~m}$ telescope in Perth also observed in $I$ band. Of the observing sites around longitude zero, the event was imaged from the $1 \mathrm{~m}$ telescope at the South African Astronomical Observatory (SAAO) using an $I$-band filter and in $J, H$, and $K_{S}$ by the $1.4 \mathrm{~m}$ Infra Red Survey Facility (IRSF), also at SAAO. The $2 \mathrm{~m}$ Liverpool Telescope (LT) observed the event in SDSS- $i$ from the Canary Islands.

As darkness fell in the Americas, a number of Chilean telescopes picked up the observing baton: the SMARTS $1.3 \mathrm{~m}$ at the Cerro Tololo Interamerican Observatory (CTIO) obtained data in $V, I$, and $H$ bands with the ANDICAM camera, and the Danish $1.54 \mathrm{~m}$ used an $I$ filter. Though in the midst of commissioning the new OGLE-IV camera at the time, the $1.3 \mathrm{~m}$ Warsaw telescope also covered the event in $I$ band. ${ }^{73}$ The $1 \mathrm{~m}$ Mt. Lemmon Telescope in Arizona imaged the event in the $I$ band, and in the extreme west, the $2 \mathrm{~m}$ Faulkes Telescope North (FTN) in Hawaii used an SDSS- $i$ filter to complete the $24 \mathrm{hr}$ coverage of the event from the Pacific. Table 1 summarizes the data obtained, which are plotted in Figure 1.

The high density of Galactic bulge star fields and the consequent degree of overlap (or blending) in stellar point-spread functions (PSF) have long since made difference image analysis (DIA) the photometry method of choice among microlensing teams. Both MOA and OGLE make their photometry available to the community, automatically reducing their data with their custom pipelines described, respectively, in Bond et al. (2001) and Udalski et al. (2003). The RoboNet data (from FTN, FTS,

\footnotetext{
73 www.ogle.astrouw.edu.pl
}

and the LT) were reduced with the project's automated data reduction pipeline, which is based around the DanDIA package (Bramich 2008). This software was also later used to reduce data from Canopus, the Perth $0.6 \mathrm{~m}$, the SAAO $1 \mathrm{~m}$, and the $H$-band data from CTIO, while the DIAPL package was used to process the images from the Danish telescope. The PLANET team released their photometry (produced by the WISIS pipeline) in real time via their Web site, and the Pysis DIA pipeline (Albrow et al. 2009) was used for later re-reduction of these data sets.

\section{VARIABILITY OF THE SOURCE STAR}

MOA-2010-BLG-073 was present in the fields of the OGLE-II and OGLE-III surveys, so the source star's I-band photometric record extends from 1998 to 2006 (Figure 2). OGLEIV was in the commissioning phase when this event took place. From this excellent baseline it was immediately clear that the source is variable over many-month timescales. This raised the possibility that shorter-term variability might obfuscate the microlensing signal, making it difficult to determine its properties.

To investigate this possibility, we performed a search for periodicities in the baseline OGLE-II and OGLE-III data, excluding the lensing event, using the ANOVA algorithm (Schwarzenberg-Czerny 1996). Due to the seasonal gaps in the baseline, we analyzed the OGLE-II data in yearly subsets as it is the best sampled, searching for periods between $P=0.5$ and 200 days. As Figure 3 demonstrates, there are no significant or persistent periodicities, other than the expected integer multiples of the 1 day sampling alias.

We then combined the OGLE-II and III data sets in order to search for periods up to $P=4000$ days. Figure 2 indicates a slight ( $\sim 0.04 \mathrm{mag}$ ) magnitude offset between the OGLE-II and 

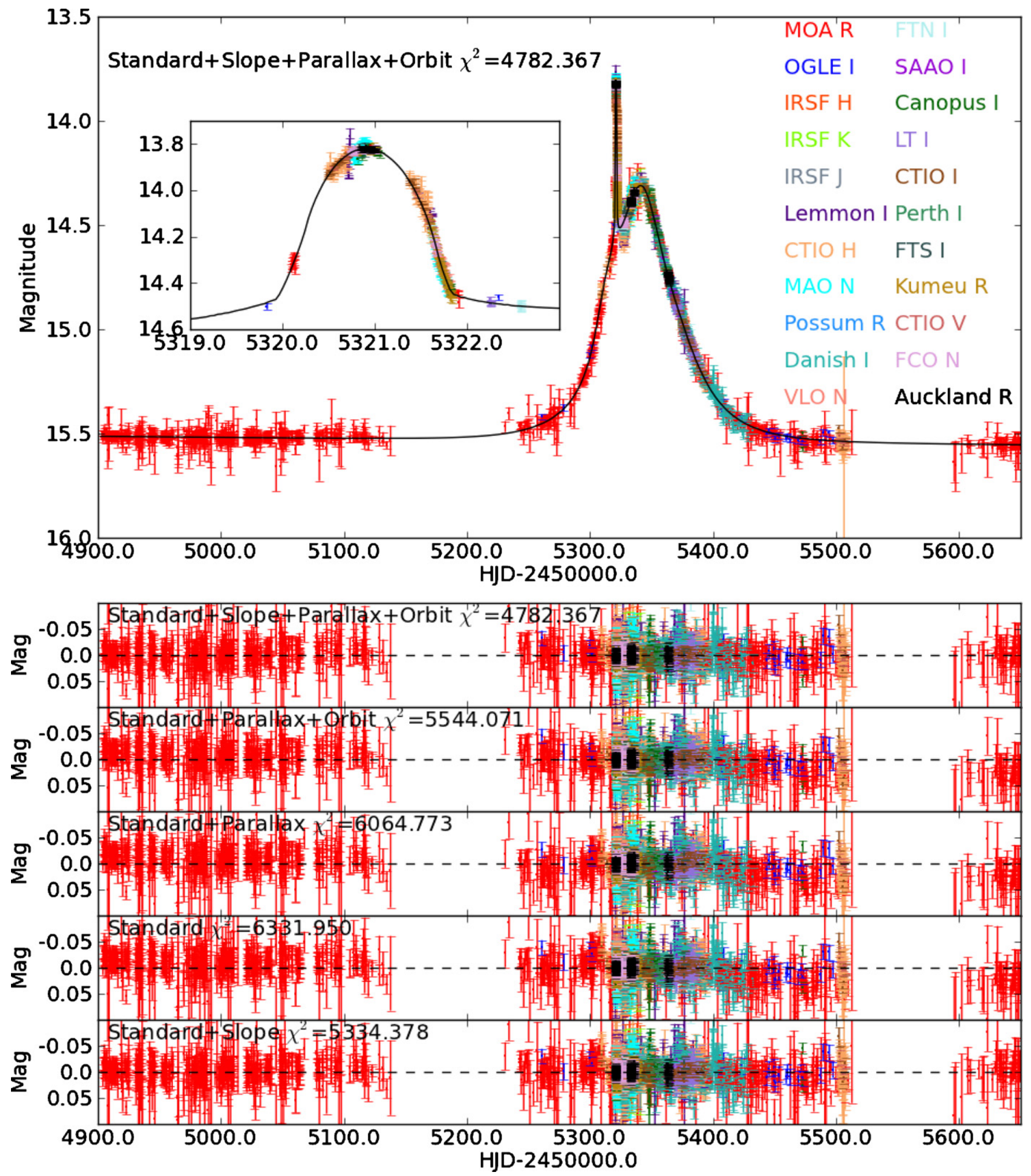

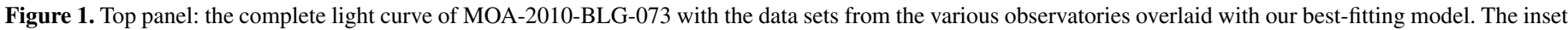
shows the anomaly in greater detail. Lower panels: the residuals of the fit for each of the best-fitting models of each class.

III data. This can occur as a residual of OGLE's photometric calibration between the two surveys, but it might also be the result of the intrinsic stellar variation. Therefore, we performed a search for periods between 0.5 and 4000 days based on the combined data both with and without this offset (estimated visually). In both cases, the periodogram was dominated by the window function; the only significant power was found in the peaks marking multiples of the 1 day alias, plus one peak at extreme low frequency corresponding to the finite length of the data set. We conclude that this star is an irregular, long-term variable, most likely as a result of pulsations.

However, there remained the possibility that the star could be irregularly variable on timescales comparable to that of the lensing event. To test this possibility, we binned the OGLE-II 


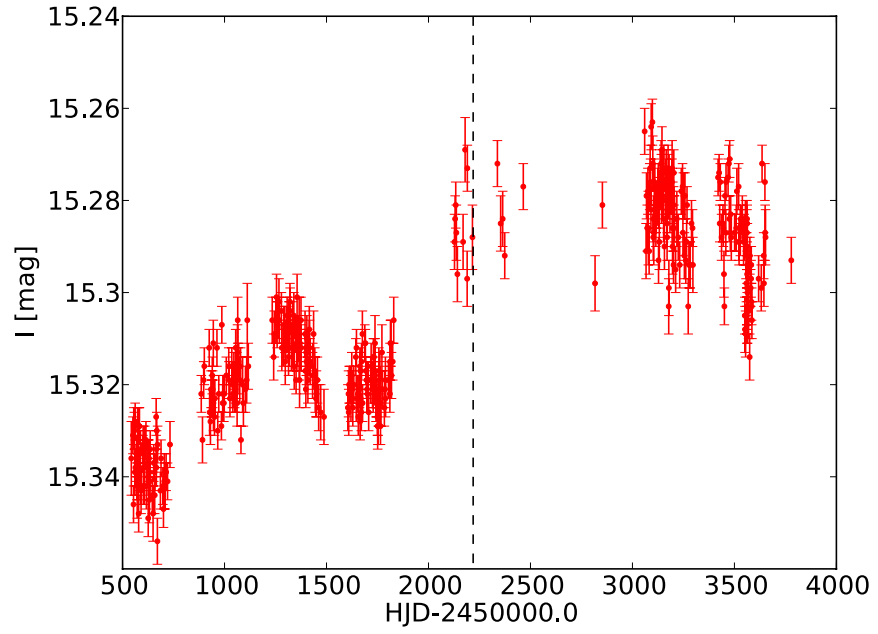

Figure 2. All available OGLE-II (1998-2001) and OGLE-III (2001 June to 2006) light-curve data for the source star of MOA-2010-BLG-073, taken prior to the event. The instrument upgrade to OGLE-III occurred around HJD = $2,452,000.0$. The apparent offset in magnitude at this time may be due in part to the difficulties of accurately calibrating photometry between the two wide-field surveys.

(A color version of this figure is available in the online journal.)
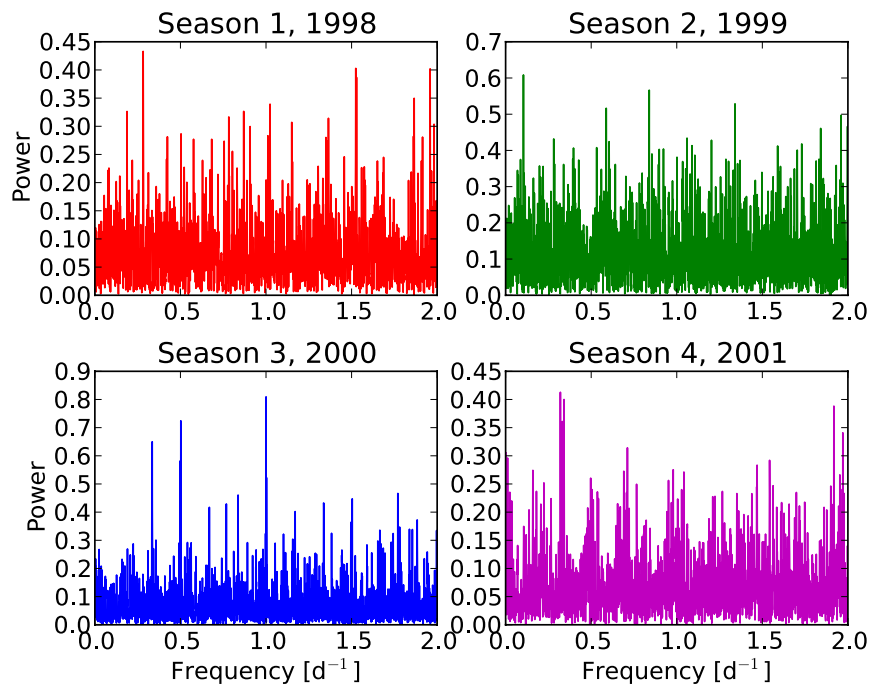

Figure 3. Periodograms of the OGLE-II (1998-2001) seasons of data, analyzed separately with the ANOVA algorithm. No significant periodicities were found in the range $P=0.5-200$ days, aside from the expected aliases.

(A color version of this figure is available in the online journal.)

light curve on a range of timescales between 2 and 1200 days. To each bin we fitted two functions, one of constant brightness and one with a linear slope, and calculated the weighted rms photometric scatter around each function per bin. We plot the average and maximum rms (calculated over all bins in the light curve) against the width of the bins in time in Figure 4. On timescales shorter than 200 days the rms scatter in the binned light curve is reasonably constant, implying no significant shortterm variability. The longer-term trend becomes clearly evident in the constant brightness curves for timescales longer than 400 days. We note that for bin widths between $\sim 800$ and 1200 days, this curve has an rms actually exceeding that of the whole light curve; this is because these bins were sufficiently wide that the first bin included the majority of the data and the most variable sections of the light curve. As the bin widths became longer, they included more data points from

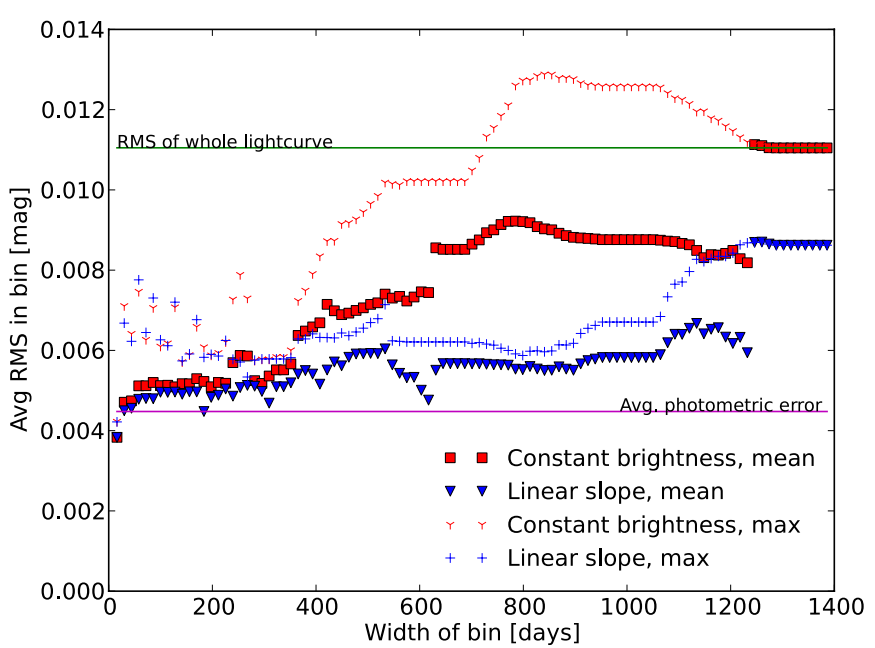

Figure 4. OGLE-II photometry was binned using a series of bins of varying width in time. The data in each bin were fitted with two functions, (1) constant brightness (2) with a linear slope, and the rms around these curves was averaged over all the bins in the light curve. The average and maximum rms for each binning is plotted here against the width of the bin, indicating variability over different timescales.

(A color version of this figure is available in the online journal.)

the relatively stable section toward the end of the OGLE-II data set, and the rms drops. The deviation of the OGLE light curve from a constant brightness exceeds $3 \sigma$ for timescales longer than $\sim 750$ days. However, it deviates from a linear slope by $\leqslant 1.6 \sigma$ for timescales less than 1000 days, so we represent this variation as a gradient in the light curve over the duration of the event.

The most intuitive way to account for the variation of the source was to measure the gradient of the light curve taken at baseline immediately before and after the event. Unfortunately, only one of the available data sets covered these periods. Fitting a straight-line model (via a nonlinear least-squares Marquardt-Levenberg algorithm) to the MOA 2009 and 2011

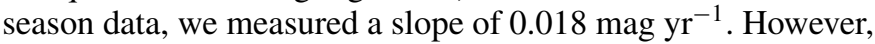
the rms scatter in the residuals of this fit was 0.016 mag, making it difficult to properly determine the slope. Additionally, in order to remove this slope from the other data sets, it would be necessary to transform the fluxes measured by each telescope onto the same scale as the MOA data. We attempted this via a linear regression approach but found that significant residuals remained. These contributed to overall higher $\chi^{2}$ values when the corrected data were fit with binary lensing models. We therefore adopted the alternative method of incorporating the slope as an additional parameter in our lensing model that we fit to the original, uncorrected data, and we describe this approach in the following sections.

\section{ANALYSIS}

In the analysis of this event, we used the established modeling software developed by S. Dong and C. Han (Dong et al. 2006; Shin et al. 2012a).

\subsection{Initial Parameters}

As a starting point for our analysis, we needed approximate values for the three parameters of the standard model for a PSPL event (not yet including the slope; this is discussed in Section 4.8): $t_{0}$, the time of peak magnification occurring at the closest projected separation between the lens and source, $u_{0}$, 
and the Einstein radius crossing time, $t_{\mathrm{E}}$. Following standard convention, all distances are quoted in units of the angular Einstein radius, $\theta_{\mathrm{E}}$, of the lens.

To estimate these parameters, we combined all available data sets into a single light curve using the following scaling to take account of the varying degrees of PSF blending from different instruments:

$$
f(t, k)=A(t) f_{s}(k)+f_{b}(k),
$$

where $f(t, k)$ is the measured flux of the target at time $t$ from data set $k, A(t)$ is the lensing magnification at that time, $f_{s}(k)$ is the flux of the source star, and $f_{b}(k)$ represents the flux of all stars blended with the source in the data set. A regression fit was used to measure $f_{s}$ and $f_{b}$ for each data set, producing an aligned light curve. Although the resulting parameter estimates are somewhat different from their "true" values due to the existence of the anomalous deviation on the light curve, they provided a starting point in parameter space.

We note that two additional parameters can contribute to a PSPL model. They are the lens parallax parameters $\pi_{\mathrm{E}, N}$ and $\pi_{\mathrm{E}, E}$ that account for the light-curve deviation caused by the motion of Earth in its orbit over the course of the event. The vector microlens parallax $\pi_{\mathrm{E}}=\mathrm{AU} / \widetilde{r_{\mathrm{E}}}$, where $\widetilde{r_{\mathrm{E}}}$ is the Einstein radius projected onto the observer plane and

$$
\pi_{\mathrm{E}} \equiv\left(\pi_{\mathrm{E}, N}, \pi_{\mathrm{E}, E}\right) \equiv\left(\cos \phi_{\pi}, \sin \phi_{\pi}\right) \pi_{\mathrm{E}},
$$

where $\phi_{\pi}$ represents the direction of lens motion relative to the source as a counterclockwise angle, north through east. However, this is generally significant only for long-timescale $(\sim$ months) events and was included at a later stage (see Section 4.6).

\subsection{Finite Source}

The sharp spike feature in the light curve is indicative of the source closely approaching or crossing a caustic. In these circumstances, it cannot be approximated by a point light source and must be treated as a disk of finite angular radius, $\rho$, with wavelength-dependent limb darkening. This is addressed within our software using the ray-shooting approach (Kayser et al. 1986): the path of light rays is traced from the image plane back to the source, taking into account the bending of the trajectory according to the lens equation. If a ray is found to "land" within the radius of the source, its intensity is computed taking limb darkening into account. We derived this from the linear limbdarkening law:

$$
I_{\lambda}(\cos \phi)=I_{\lambda}(1)\left[1-u_{\lambda}(1-\cos \phi)\right],
$$

where $I_{\lambda}$ is the intensity of the source at radius $\phi$ from the center, relative to the central intensity $I_{\lambda}(1)$ in the same wavelength, $\lambda$, scaled by the coefficient $u_{\lambda}$. While more accurate limbdarkening models are available, they are not commonly used in microlensing analyses due to the complexity introduced by combining data from many sources (Bachelet et al. 2012 discussed this in more detail). The values of $u_{\lambda}$ for each passband were calculated from the Kurucz ATLAS9 stellar atmosphere models presented by Kurucz (1979) using the method of Heyrovský (2007). However, within the microlensing community and software, Equation (3) more commonly follows the formalism derived by Albrow et al. (1999):

$$
I_{\lambda}=\frac{F_{\lambda}}{\pi \theta_{*}^{2}}\left[1-\Gamma_{\lambda}\left(1-\frac{3}{2} \cos \phi\right)\right],
$$

where $F_{\lambda}$ is the total flux from the source in a given passband and $\phi$ is the angle between the line of sight to the observer and the normal to the stellar surface. The limb-darkening coefficient, $\Gamma_{\lambda}$, is related to $u_{\lambda}$ by

$$
\Gamma_{\lambda}=\frac{2 u_{\lambda}}{3-u_{\lambda}} .
$$

The values of $u_{\lambda}$ and $\Gamma_{\lambda}$ applied for each data set are presented in Table 1. The lensing magnification is then computed as the ratio of the number of rays reaching the source plane relative to the number in the image plane. This approach is only required while the source is close to the caustic. At larger separations, the software employs a semi-analytic hexadecapole approximation to the finite source calculation to improve computation speeds (Pejcha \& Heyrovský 2009; Gould 2008).

\subsection{Standard Binary Model Grid Search}

To model the light curve of a binary lens event, we introduced three additional parameters: $q=M_{L, 2} / M_{L, 1}$, the ratio of the masses of the two bodies composing the lens, where $M_{L, 1}$ is the more massive component; $s_{0}$, the projected separation of those masses; and $\alpha_{0}$, the angle of the trajectory of the lensed source star, relative to the lens's binary axis. The frame of reference was defined to be at rest with respect to Earth at time $t_{0, \mathrm{par}}$, which we took to be the time of caustic crossing at HJD = $2,455,321.0$, estimated from the easily identifiable feature in the light curve (following the notation of Skowron et al. 2011).

With seven variables in the model $\left(t_{0}, u_{0}, t_{\mathrm{E}}, \alpha_{0}, s_{0}, q, \rho\right)$, a number of different lens/source configurations may produce similar light curves, so it was necessary to thoroughly explore a large area of parameter space in order to ensure that all possible solutions are identified. We therefore constructed a grid of models, spanning set ranges in the values of the three variables upon which the overall $\chi^{2}$ of the fit depended most sensitively, $s_{0}, q$, and $\alpha_{0}$. Each node in this grid took fixed values of $\left(s_{0}, q, \alpha_{0}\right)$ and used a Markov Chain Monte Carlo (MCMC) approach (Dong et al. 2006) to find the best-fitting model by optimizing the other parameters. To improve efficiency, a magnification map is generated by ray shooting for each point in the grid from which the model light curves used to compute the $\chi^{2}$ are drawn. The grid covered the following range: $\log \left(s_{0}\right)=-0.6: 0.6$ in steps of $0.012, \log (q)=-4.0: 1.0$ in steps of 0.05 , and $\alpha=0.0: 6.3$ in steps of 0.6 .

Mapping out the $\chi^{2}$ for each node in this grid, we found a number of local minima. Visual inspection of these models overlaid on the light curve demonstrated that some more closely followed the data than others. Our first pass analysis included substantial baseline photometry before and after the event. This was not well fit by the models due to the variability of the source, and hence the $\chi^{2}$ map gave a distorted view of regions in parameter space that best match the event. For this reason, we proceeded by repeating the grid search using just data taken during 2010. This produced two clear minima in $\chi^{2}$, of which one model stood out as by far the best match to the data. We then conducted a refined grid search over this restricted region of parameter space, taking smaller incremental steps.

\subsection{Optimized Standard Binary Model}

The refined grid search produced a reasonable model, fitting the majority of the data from all telescopes. This was used as a guide to identifying likely outlying data points, for which the quality of the reduction was then checked. A handful of data 
Table 2

The $\chi^{2}$ for the Best-fitting Model in Each Class, Comparing $u_{0}>0$ and $u_{0}<0$ Solutions

\begin{tabular}{lcc}
\hline \hline Model & \multicolumn{2}{c}{$\chi^{2}$} \\
\cline { 2 - 3 } & $u_{0}>0$ & $u_{0}<0$ \\
\hline Standard & 6331.950 & 6331.950 \\
Standard+Parallax & 6064.773 & 6099.487 \\
Standard+Parallax+Orbital Motion & 5544.071 & 5690.003 \\
Standard+Parallax+Orbital Motion with Slope & 4782.367 & 4802.606 \\
Standard+Slope & 5334.378 & 5334.378 \\
\hline
\end{tabular}

points were removed at this stage. However, this model included only fixed values for $s_{0}, q$, and $\alpha_{0}$. To properly determine the standard binary model for this event, our next step was to allow the seven parameters $\left(t_{0}, u_{0}, t_{\mathrm{E}}, \alpha_{0}, s_{0}, q, \rho\right)$ to be optimized during the MCMC fitting process, which used the grid search results as its starting point. At this point we included the extended baseline data from MOA for seasons 2009 and 2011, as these fall within the period for which the source's variability can be approximated with a straight line; we address this in Section 4.8 .

\subsection{Normalization of Photometric Errors}

When fitting microlensing events, the reduced $\chi^{2}$ of the fit on a per data set basis, $\chi_{\text {red, }}^{2}$, typically produces a range of values both less than and exceeding the expected unity value. This can occur as different groups have slightly different ways of estimating photometric errors, but it can lead to over- or underemphasis being placed on particular data sets during the modeling process.

A common technique to address this issue is to arrive at a complete model for the event and then use this model to renormalize the original photometric errors of each data set, $e_{\text {orig }}$, according to the expression

$$
e_{\text {new }}=a_{0} \sqrt{e_{\text {orig }}^{2}+a_{1}^{2}}
$$

We first conducted the sequence of models described in the following sections in order to find the best model for the event. We then set the coefficients $a_{0}, a_{1}$ such that the $\chi_{\text {red }}^{2}$ relative to that model equaled unity; the adopted values are given in Table 1. We then repeated our MCMC fitting process, starting with the standard binary model and systematically adding parameters in to determine the extent of improvement in the model $\chi^{2}$ in each case. We compare models in Tables 2 and 3, and we plot the residuals (data - model) in Figure 1. In the following sections, the $\chi^{2}$ values given are those post-renormalization.

\subsection{Parallax}

Given that the event's $t_{\mathrm{E}} \sim 44$ days $\sim 0.12 \mathrm{yr}$, it was necessary to include parallax in our model. Using the parameters of the standard binary model as a starting point, we allowed our fitting process to optimize for $\pi_{\mathrm{E}, E}$ and $\pi_{\mathrm{E}, N}$ also. We found that this significantly reduced the $\chi^{2}$ of the overall fit to 6064.773. By default, this procedure explored models with positive projected separations at closest approach of the lens and source, that is, $u_{0}>0$, which we define as the source's trajectory passing the

Table 3

The Best-fitting Parameters

\begin{tabular}{|c|c|c|c|c|c|}
\hline $\begin{array}{l}\text { Parameter } \\
\text { (Units) }\end{array}$ & Standard & $\begin{array}{l}\text { Standard } \\
+ \text { Slope }\end{array}$ & $\begin{array}{l}\text { Standard } \\
+ \text { Parallax }\end{array}$ & $\begin{array}{l}\text { Standard+Parallax } \\
\text { +Orbital Motion }\end{array}$ & $\begin{array}{c}\text { Standard+Parallax } \\
+ \text { Orbital Motion+Slope }\end{array}$ \\
\hline$\overline{\chi^{2}}$ & 6331.950 & 5334.378 & 6064.773 & 5544.071 & 4782.367 \\
\hline$\Delta \chi^{2 \mathrm{a}}$ & 1549.583 & 552.011 & 1282.406 & 761.704 & 0.0 \\
\hline \multirow[t]{2}{*}{$t_{0}\left(\mathrm{HJD}^{\prime}\right)^{\mathrm{b}}$} & 5344.32 & 5344.38 & 5344.47 & 5344.83 & 5344.69 \\
\hline & \pm 0.01 & \pm 0.01 & \pm 0.02 & \pm 0.03 & \pm 0.02 \\
\hline \multirow[t]{2}{*}{$u_{0}$} & 0.4089 & 0.403 & 0.403 & 0.381 & 0.386 \\
\hline & \pm 0.0009 & \pm 0.001 & \pm 0.001 & \pm 0.001 & \pm 0.001 \\
\hline \multirow[t]{2}{*}{$t_{\mathrm{E}}$ (days) } & 43.82 & 44.84 & 43.49 & 43.4 & 44.3 \\
\hline & \pm 0.08 & \pm 0.09 & \pm 0.09 & \pm 0.1 & \pm 0.1 \\
\hline \multirow{2}{*}{$s_{0}$} & 0.7692 & 0.7725 & 0.7717 & 0.7792 & 0.7750 \\
\hline & \pm 0.0005 & \pm 0.0005 & \pm 0.0006 & \pm 0.0007 & \pm 0.0007 \\
\hline \multirow[t]{2}{*}{$q$} & 0.0705 & 0.0677 & 0.0695 & 0.0683 & 0.0654 \\
\hline & \pm 0.0005 & \pm 0.0005 & \pm 0.0006 & \pm 0.0006 & \pm 0.0006 \\
\hline \multirow[t]{2}{*}{$\alpha_{0}$} & 0.180 & 0.171 & 0.198 & 0.297 & 0.221 \\
\hline & \pm 0.003 & \pm 0.003 & \pm 0.003 & \pm 0.006 & \pm 0.007 \\
\hline \multirow[t]{2}{*}{$\rho$} & 0.01963 & 0.01912 & 0.01931 & 0.0163 & 0.0165 \\
\hline & \pm 0.00006 & \pm 0.00007 & \pm 0.00008 & \pm 0.0001 & \pm 0.0001 \\
\hline \multirow[t]{2}{*}{$\pi_{\mathrm{E}, N}$} & & & 0.18 & 0.96 & 0.37 \\
\hline & & & \pm 0.02 & \pm 0.04 & \pm 0.05 \\
\hline \multirow[t]{2}{*}{$\pi_{\mathrm{E}, E}$} & & & -0.124 & 0.09 & 0.01 \\
\hline & & & \pm 0.007 & \pm 0.01 & \pm 0.01 \\
\hline \multirow[t]{2}{*}{$d s / d t\left(\mathrm{yr}^{-1}\right)$} & & & & 0.53 & 0.49 \\
\hline & & & & \pm 0.02 & \pm 0.02 \\
\hline \multirow[t]{2}{*}{$d \alpha / d t\left(\mathrm{yr}^{-1}\right)$} & & & & -1.21 & -0.37 \\
\hline & & & & \pm 0.06 & \pm 0.08 \\
\hline \multirow[t]{2}{*}{ 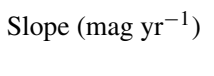 } & & -0.0160 & & & -0.0153 \\
\hline & & \pm 0.0004 & & & \pm 0.0004 \\
\hline
\end{tabular}

Notes.

a Improvement in $\chi^{2}$ relative to that of the best-fitting model.

b All timestamps are abbreviated to $\mathrm{HJD}^{\prime}=\mathrm{HJD}-2,450,000.0$. 
caustic at positive values of $\theta_{y}$ in the lens plane (see Figure 6). For the standard model case the symmetry with respect to the binary axis of the caustic means that the $u_{0}<0$ solutions are identical. However, once parallax was included, this was no longer the case, so we also explored $u_{0}<0$ solutions (this degeneracy is further discussed in Park et al. 2004). The parameters of the $u_{0}>0$ model were taken as a starting point for the fit, except that the sign of $u_{0}$ was reversed and the $\alpha_{0}$ value became $2 \pi-\alpha_{0}$. We found the best-fitting $u_{0}<0$ model to be slightly less favored, with $\chi^{2}=6099.487$.

\subsection{Lens Orbital Motion}

The mass ratio and projected separation determined from this model put this event close to the boundary between close and intermediate/resonant caustic structure. In this regime, small changes in the projected separation of the lensing bodies due to their orbital motion can effectively change the shape of the caustic (see Figure 6) while the event is underway, sometimes causing detectable deviations in the light curve. To explore this possibility, we included additional parameters in our model to describe the change in projected binary separation, $d s / d t$, and the rate of change of the angle of the projected binary axis, $d \alpha / d t$. Again we found that the $u_{0}>0$ model gave the best fit, with $\chi^{2}=5544.071$, compared with 5690.003 when $u_{0}<0$. This type of orbital motion is classified as "separational" in the schema put forward by Penny et al. (2010) and is detected in this event as the source happens to cross the cusp of the caustic in the position where the caustic changes most rapidly.

\subsection{Sloping Baseline}

While taking these second-order effects into account significantly improved the fit to the data, the overall $\chi^{2}$ remained rather high. Visual inspection of the light curve still showed a gradient, especially in the 2010 baseline before, relative to after, the event. Based on our analysis in Section 3, this trend is likely to be part of the longer-term variability of the source and not associated with the microlens. In order to determine the true lens/source characteristics, though, this trend had to be taken into account.

The OGLE-II and III data demonstrate that the source variation over the $\sim 150$ day timescale of the event can be approximated by a straight line, rather than a higher-order function. We therefore introduced a "slope" parameter to our model, representing the linear rate of change in magnitude during the event. This further improved the $\chi^{2}$, and the bestfitting model was once again the $u_{0}>0$ solution with $\chi^{2}=$ 4782.367 .

We note that there exist degeneracies between the slope parameter and those for parallax and orbital motion as they can be used to fit similar residuals in the light curve. To test for possible degeneracies, we also fit a standard model plus the slope parameter alone and found that $\chi^{2}=5334.378$. The value for the slope from this model, $-0.0160 \pm 0.0004$, was consistent with that derived from the model including parallax and orbital motion, $-0.0153 \pm 0.0004$.

\subsection{Second-order Effects}

With the slope parameter included, we had accounted for all the physical effects that we expected to be present in the light curve. Having found that the residuals showed no further variation at a level detectable above the photometric noise, we did not attempt to include second-order effects such as xallarap, etc.

\subsection{Final Model}

All our models are compared in Table 2, and the parameters of the best-fitting models are presented in Table 3 and Figure 1. We plot the $\chi^{2}$ for each link in the MCMC chain for all parameters against one another in the best-fitting model in Figure 5. This plot was used as a diagnostic throughout the fitting process, as any correlations between parameters display distinct trends as the chain moves toward the minimum. The caustic structure changed during the course of the event, so in Figure 6 we show the structure at two distinct times: the first at the time of the first caustic crossing during the anomaly, and the second at the time of closest approach.

\section{PHYSICAL PARAMETERS}

The purpose of this model is to ultimately arrive at the physical parameters of the lens and source, which can be achieved using the known relations between these and the lensing parameters obtained from the modeling.

Chiefly of interest is the mass of the lensing system, $M_{L \text {,tot }}$, which can be determined explicitly for events where parallax is measurable provided that the angular extent of the Einstein radius, $\theta_{\mathrm{E}}$, is known from

$$
M_{L, \mathrm{tot}}=\frac{c^{2}}{4 G} \widetilde{r}_{\mathrm{E}} \theta_{\mathrm{E}}=\frac{c^{2} \mathrm{AU}}{4 G} \frac{\theta_{\mathrm{E}}}{\pi_{\mathrm{E}}},
$$

where $\widetilde{r}_{\mathrm{E}}$ is the Einstein radius projected from the source onto the observer's plane. The model parameter $\rho$ represents the angular size of the source star $\theta_{S}$ in units of the angular Einstein radius $\theta_{\mathrm{E}}$. We derive this from the crossing time taken for the source to travel behind the lens, $t_{*}=\mu \theta_{S}$, where $\mu$ is the relative source-lens proper motion. $\rho$ can then be written as

$$
\rho=\frac{t_{*}}{t_{\mathrm{E}}}=\frac{\theta_{S}}{\theta_{\mathrm{E}}} .
$$

These parameters also yield the distance to the lens,

$$
\frac{\mathrm{AU}}{D_{L}} \equiv \pi_{L}=\theta_{\mathrm{E}} \pi_{\mathrm{E}}+\frac{\mathrm{AU}}{D_{S}},
$$

which in turn yields the projected separation between the lens components,

$$
a_{\perp}=s_{0} D_{L} \theta_{\mathrm{E}}
$$

and the relative proper motion between lens and source, when combined with $t_{\mathrm{E}}$,

$$
\mu=\frac{\theta_{\mathrm{E}}}{t_{\mathrm{E}}} .
$$

The appreciable lens orbital motion during this event also allows us to test whether the companion object is bound to the primary lensing mass, via the ratio of its kinetic to potential energy:

$$
\mathrm{KE} / \mathrm{PE}=\frac{\left(s_{0} R_{\mathrm{E}}\right)^{3} \gamma^{2}}{8 \pi^{2} M_{L}},
$$

where $\gamma$ relates the two lens orbital parameters, $\gamma^{2}=$ $\left(d s / d t / s_{0}\right)^{2}+(d \alpha / d t)^{2}$, and where the masses are in units of $M_{\odot}$, the distances in $\mathrm{AU}$, and time measured in years.

However, these expressions include two key terms that are as yet unknown: $\theta_{S}$, the angular source radius, and $D_{S}$, the distance to the source. In order to extract the physical characteristics of the lens, we therefore turned our attention to the characteristics of the source. 


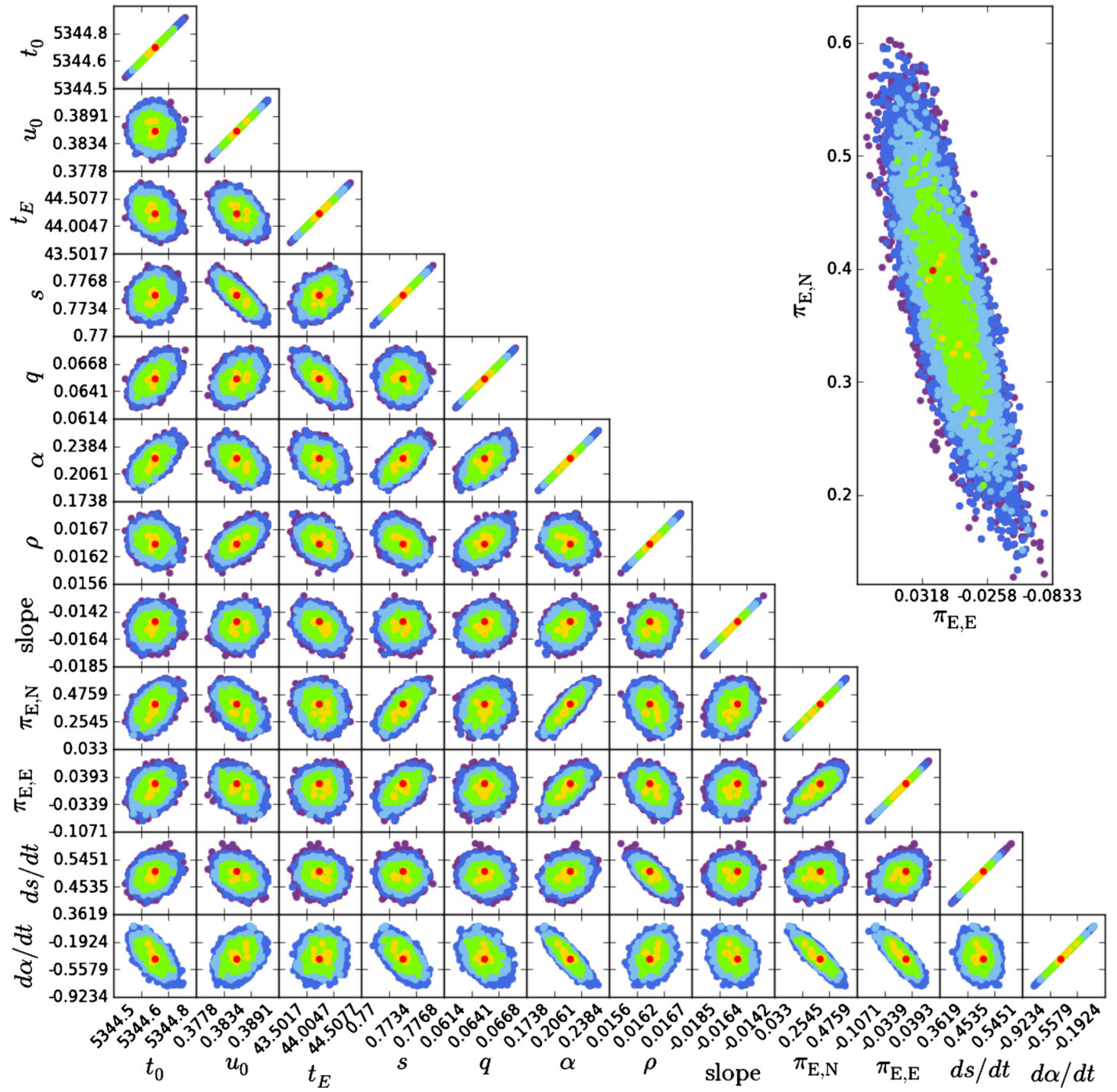

Figure 5. Chi-squared contours plotted as a function of the parameters fitted in the MCMC fit for the best model. The red, orange, green, light blue, dark blue, and purple colors indicate the regions with $\Delta \chi^{2}<1 \sigma-6 \sigma$ (respectively) from the best-fit solution. Inset: close-up of the contours for the parallax parameters.

\subsection{Source Star}

Long-exposure $V, I$ images were acquired by the CTIO $1.3 \mathrm{~m}$ at several epochs, which enabled us to plot the color-magnitude diagram for the field including the source star (Figure 7). By observing the event at different levels of lensing magnification, these data can be incorporated into the model that yields the source and blended light fluxes, $f_{s}, f_{b}$ for those data, and hence the instrumental magnitudes and colors of the source and blend. But we note that these uncalibrated fluxes also suffer from the high degree of extinction along the line of sight to the Galactic bulge. To calculate the dereddened color,
$(V-I)_{S, 0}$, and magnitude, $I_{S, 0}$, of the source, we needed to calibrate the instrumental fluxes $f_{s}$ and $f_{b}$ relative to a standard candle.

Figure 7 clearly shows a locus of stars centered at $I_{\mathrm{RC} \text {,inst }}=$ $15.821 \pm 0.05 \mathrm{mag},(V-I)_{\mathrm{RC} \text {,inst }}=-0.350 \pm 0.05 \mathrm{mag}$. This consists of a clump of red giant stars, for which stellar theory predicts a stable absolute luminosity, varying only slightly with age and chemical composition. Their frequent occurrence makes these objects useful as standard candles. Stanek et al. (1998) established photometric calibrations for red clump magnitudes, which were later refined by Alves et al. (2002) using Hipparcos data. Most recently, Nataf et al. (2012) were able to measure 


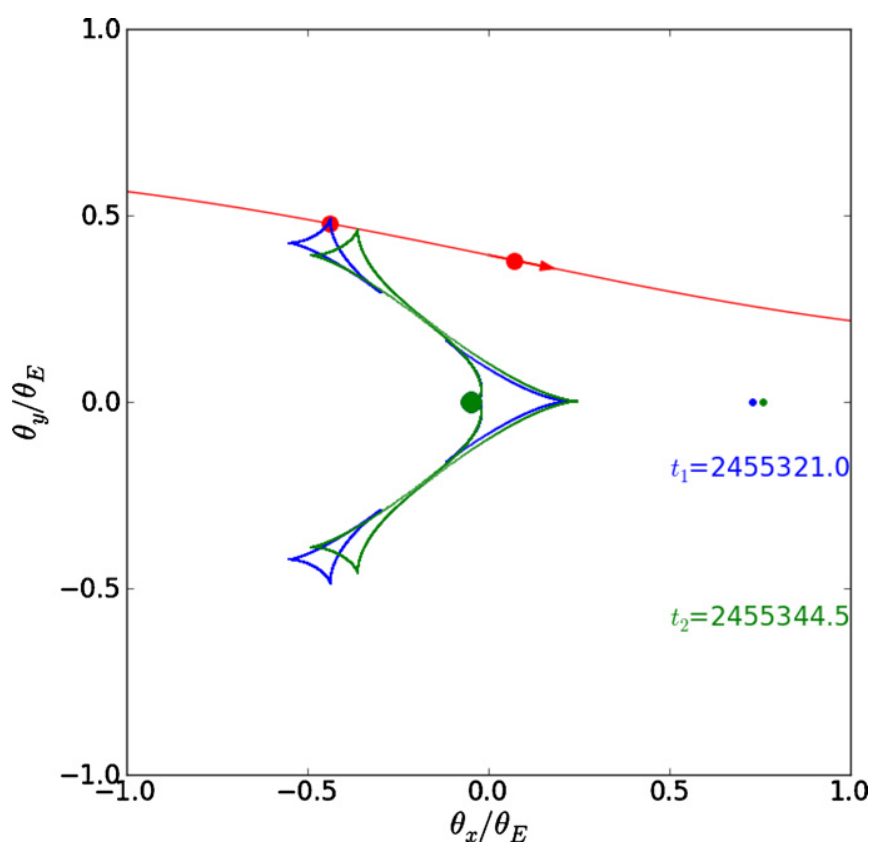

Figure 6. Maps of the caustic structure during the anomaly and at the time of closest approach. The red line and arrow indicate the trajectory of the source in a reference frame centered on the barycenter of the lensing system, while the red dots indicate the position of the source at these times. The green and blue dots indicate the positions of $M_{L, 1}$ (largest) and $M_{L, 2}$ at both times (radii of dots not to scale).

(A color version of this figure is available in the online journal.)

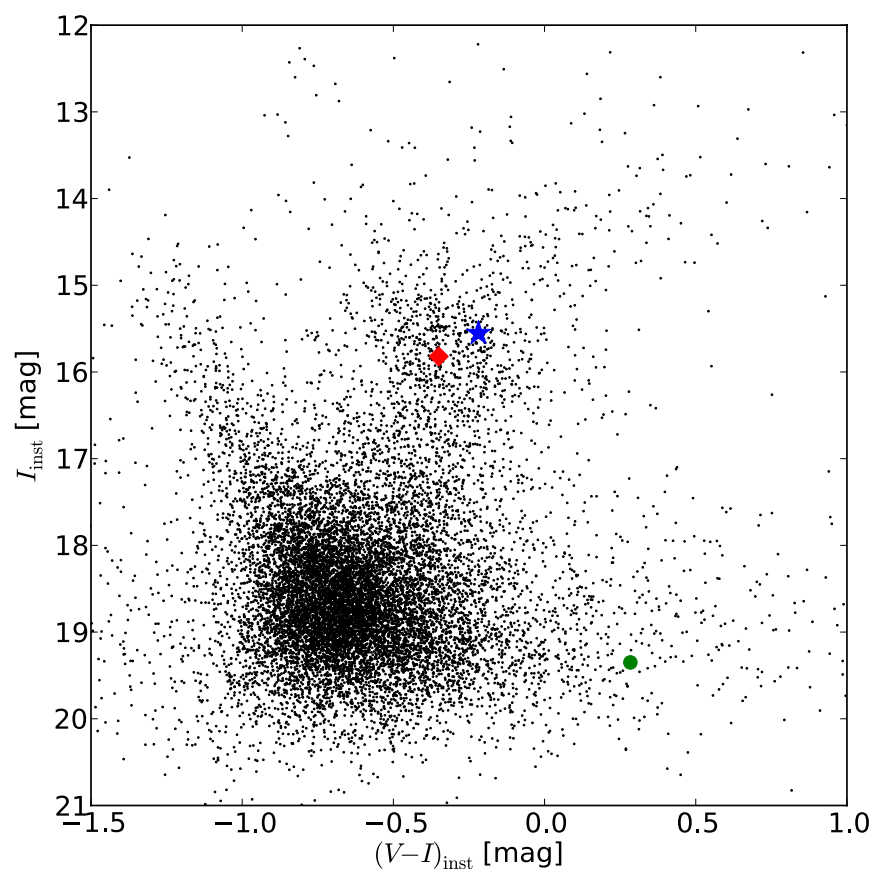

Figure 7. Instrumental color-magnitude diagram for the field of view including the lensing source star. The position of the source is marked with a blue star, relative to the center of the red giant clump highlighted with a red diamond. The green circle indicates the color and $V$ magnitude of light blended in the CTIO photometry.

(A color version of this figure is available in the online journal.)

the dereddened apparent magnitude of the red clump stars at the Galactocentric distance, $I_{\mathrm{RC}, 0}=14.443$. By mapping the distances, $D_{\mathrm{RC}}$, to red clump stars in the Galactic bar as a function of Galactic longitude, $l$, they found an apparent viewing
Table 4

The Physical Parameters of the Lensing System and Source Star, Derived from the Model Including Slope, Parallax, and Orbital Motion, plus Color Information

\begin{tabular}{|c|c|c|}
\hline Parameter & Units & Value \\
\hline$\theta_{S}$ & $\mu$ as & $9.143 \pm 0.792$ \\
\hline$\theta_{\mathrm{E}}$ & mas & $0.557 \pm 0.09$ \\
\hline$R_{S}$ & $R_{\odot}$ & $14.7 \pm 1.3$ \\
\hline$M_{L, 1}$ & $M_{\odot}$ & $0.16 \pm 0.03$ \\
\hline$M_{L, 2}$ & $M_{\mathrm{J}}$ & $11.0 \pm 2.0$ \\
\hline$M_{L, \text { tot }}$ & $M_{\odot}$ & $0.17 \pm 0.03$ \\
\hline$D_{L}$ & $\mathrm{kpc}$ & $2.8 \pm 0.4$ \\
\hline$a_{\perp}$ & $\mathrm{AU}$ & $1.21 \pm 0.16$ \\
\hline $\mathrm{KE} / \mathrm{PE}$ & & 0.079 \\
\hline Proper motion & mas $\mathrm{yr}^{-1}$ & $4.60 \pm 0.4$ \\
\hline$I_{S, \text { inst }}$ & mag & $15.554 \pm 0.007$ \\
\hline$V_{S, \text { inst }}$ & mag & $15.335 \pm 0.007$ \\
\hline$(V-I)_{S \text {,inst }}$ & mag & $-0.22 \pm 0.01$ \\
\hline$I_{\mathrm{RC}, \text { inst }}$ & mag & $15.821 \pm 0.05$ \\
\hline$(V-I)_{\mathrm{RC}, \text { inst }}$ & mag & $-0.350 \pm 0.05$ \\
\hline$I_{\mathrm{RC}, 0}$ & mag & 14.443 \\
\hline$(V-I)_{\mathrm{RC}, 0}$ & mag & 1.09 \\
\hline$I_{S, 0}$ & mag & 13.976 \\
\hline$V_{S, 0}$ & mag & 15.197 \\
\hline$(V-I)_{S, 0}$ & mag & $1.221 \pm 0.051$ \\
\hline$(V-K)_{S, 0}$ & mag & 2.852 \\
\hline$K_{S, 0}$ & mag & 12.345 \\
\hline$J$ (2MASS) & mag & $13.686 \pm 0.053$ \\
\hline$H$ (2MASS) & mag & $12.926 \pm 0.057$ \\
\hline$K_{S}(2 \mathrm{MASS})$ & mag & $12.642 \pm 0.054$ \\
\hline
\end{tabular}

angle on the bar of $\phi_{\mathrm{Bar}}=40^{\circ}$,

$$
\frac{R_{0}}{D_{\mathrm{RC}}}=\frac{\sin \phi+l}{\phi}=\cos l+\sin l \cot \phi,
$$

where Nataf et al. (2012) measured $R_{0}$ to be $8.20 \mathrm{kpc}$. For the field of MOA-2010-BLG-073 (Galactic coordinates: $l=$ $4.81030, b=-3.50131)$, we derive $D_{\mathrm{RC}}=7.48 \mathrm{kpc}$, and we assumed that the source star lies behind the same amount of dust as the red clump stars and at the same distance. Scaling the dereddened apparent magnitude of the red clump stars, $I_{\mathrm{RC}, 0}$, appropriately for the slightly closer distance of the stars in this field, $I_{\mathrm{RC}, \mathrm{app}}=I_{\mathrm{RC}, 0}+\Delta I$, where

$$
\Delta I=5 \log _{10} R_{0} / D_{\mathrm{RC}} .
$$

We found $\Delta I=0.20 \mathrm{mag}$, and so the distance modulus to the red clump and the source in this field is $I_{\mathrm{RC}, \text { app }}=14.24 \mathrm{mag}$. Bensby et al. (2011) determined the intrinsic $(V-I)_{\mathrm{RC}, 0}=$ 1.09 mag for red clump stars, so we were able to derive their absolute $V$ magnitude of $M_{V, \mathrm{RC}, O}=0.97 \mathrm{mag}$. Combining these results with the measured $\Delta(V-I)_{\text {inst }}$ and $\Delta V_{\text {inst }}$ between the source and red clump in the CTIO data, we then derived the dereddened color $(V-I)_{S, 0}$ and magnitude $I_{S, 0}$ of the source, summarized in Table 4.

Bessell \& Brett (1988) provided a relationship between ( $V-$ $I)$ and $(V-K)$ color indices, and Kervella et al. (2004) related $(V-K)$ to angular radius for giant and dwarf stars. Having thus determined $D_{S}$ and $\theta_{S}$, we derived the physical parameters of the lensing system, which are also summarized in Table 4 . The color and large source radius of $14.7 \pm 1.3 R_{\odot}$ imply that this star is a $\mathrm{K}$-type giant, which is consistent with the observed photometric variability. Jorissen et al. (1997) found that red giant stars with spectral types later than early-K are all variable, with amplitudes 


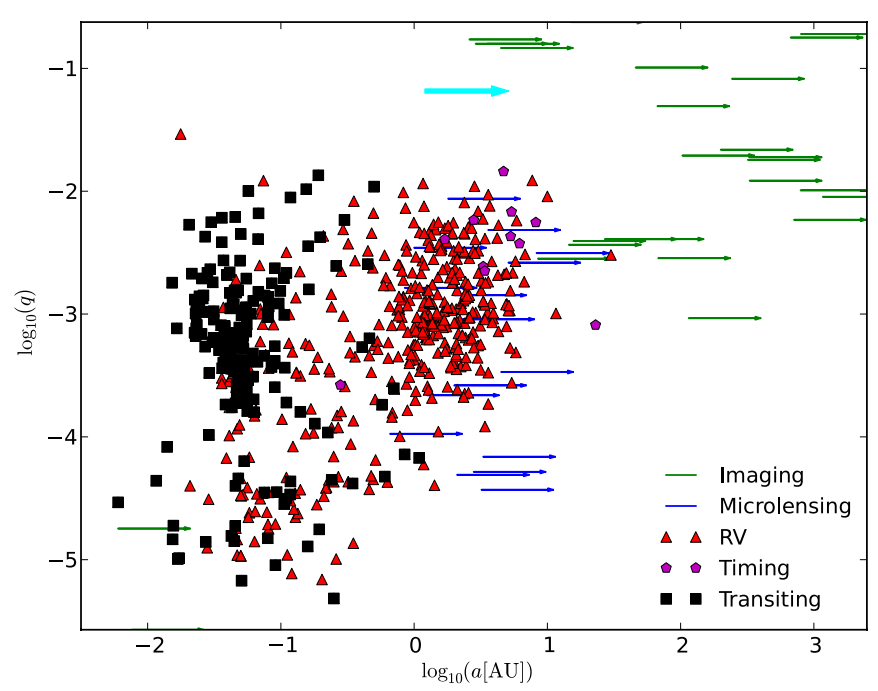

Figure 8. Ratio of planet mass to host star mass plotted against semimajor axis for all exoplanets for which these parameters are available (source: www.exoplanet.eu; Schneider et al. 2011). The true orbital semimajor axis is plotted where it has been measured; otherwise, the projected (minimum) separation has been indicated with an arrow, where the base of the arrow marks the measured value. Very few objects have been found between $\log _{10}(q)=-1$ and -2 . This corresponds to the brown dwarf desert. The location of MOA2010-BLG-073L, highlighted in cyan and bold, lies between the brown dwarf and planetary regimes.

increasing from microvariability to several magnitudes toward cooler temperatures and timescales from days to years. Kiss et al. (2006) note that irregular photometric variability may be caused by large convection cells, or may actually be the result of a number of simultaneous periodic pulsation modes, and many examples have been identified from time-domain surveys (e.g., Wray et al. 2004; Woźniak et al. 2004; Eyer \& Blake 2005; Ciechanowska et al. 2006). We note that the star was detected by the Two Micron All Sky Survey (2MASS; Skrutskie et al. 2006) as source 2MASS J18101138-2631226 with colors $(J-H)=$ $0.76 \pm 0.078 \mathrm{mag}$ and $\left(H-K_{S}\right)=0.284 \pm 0.079 \mathrm{mag}$. Although the MASS field is crowded, the star's PSF is distinct and their photometry for it has the best-quality AAA flag. These colors are consistent with a giant star and with our derived value for $K_{S, 0}=12.345 \mathrm{mag}$, when we take into account $A K_{S}=0.24 \mathrm{mag}$ from the VVV survey (Gonzalez et al. 2012).

Nataf et al. (2012) explain that their value of our viewing angle of the Galactic bar is a "soft upper bound" because the distance along the plane to the greatest density of stars along the line of sight to a triaxial bar structure is less than the distance to the structure's major axis on the far side and greater on the near side. As the physical parameters derived for MOA-2012-BLG-073 are somewhat dependent on the value of our viewing angle of the Galactic bar and Nataf et al. (2012) quote consistent results with values as low as $25^{\circ}$, we explored the potential impact of this on our results. A reduced viewing angle would produce a smaller distance to the source, changing its dereddened magnitude and color. The resulting increase in source radius produces a corresponding reduction in the value of $D_{L}$ and increases in the lens masses. However, we found that the physical parameter values do not change by more than the errors quoted in Table 4, implying that this is not the dominant source of uncertainty. Finally, we computed the physical parameters derived from the best-fitting $u_{0}<0$ model for comparison and found that the masses derived changed by $<1 \sigma$.

\section{DISCUSSION}

Earth's movement during this relatively long timescale ( $t_{\mathrm{E}}$ $=44.3$ days) microlensing event resulted in a gradual shift in our perspective on the lensing system, breaking the symmetry of the caustic. Meanwhile, the change in projected separation of the lensing objects modified the shape of the caustic just as the source's trajectory happened to pass close by. If not for these subtle variations, it can be seen from Figure 6 that a source trajectory $u_{0}>0$ would produce exactly the same light curve as a $u_{0}<0$ trajectory. As it is, for this event, the $u_{0}>0$ solution best explains our observations, though the difference in $\chi^{2}$ relative to the corresponding $u_{0}<0$ solution is very small $\left(\Delta \chi^{2}=20.2\right)$ compared with the $\chi^{2}$ of both fits.

The measurable parallax signature enables us to determine the masses of the lensing bodies. The primary lensing object has $M_{L, 1}=0.16 \pm 0.03 M_{\odot}$, making it an M-dwarf star. The companion's mass is $M_{L, 2}=11.0 \pm 2.0 M_{\mathrm{J}}$. This places it in the brown dwarf desert (see Figure 8), though we note that this traditionally refers to close-in companions, and since microlensing and direct imaging measure only the projected separation, we know only their minimum orbital semimajor radii. Regardless, it is clear that MOA-2010-BLG-073L b is close to the mass threshold for deuterium burning $\left(\sim 0.012 M_{\odot}=12.6 M_{\mathrm{J}}\right)$ quoted as the nominal boundary between planets and brown dwarfs established by the IAU (Chabrier et al. 2005). So what kind of object is it?

No further orbital or metallicity information is available for this event, which might have shed light on its evolutionary history. Theoretical isochrones predict that a star of this low mass will not have lost a significant amount of material over its lifetime, so we can say that MOA-2010-BLG-073L b formed as a high mass ratio binary. Models of protoplanetary disks have the expectation that disk mass, $M_{D}$, will scale linearly with star mass $M_{*}$ (Williams \& Cieza 2011), $M_{D} / M_{*} \sim 1 \%$ at young ages, but this would limit $M_{D}$-and hence $M_{P, \max }$ - to $\sim 1-10 M_{\mathrm{J}}$ in the case of MOA-2010-BLG-073L. So it seems questionable whether such a massive companion could have formed in a protostellar disk, via either core accretion or gravitational instability.

Bonnell et al. (2008) and Kroupa \& Bouvier (2003) discuss a number of mechanisms that can produce an M-dwarf/brown dwarf binary following gravitational fragmentation in a molecular cloud.

1. Embryo rejection model. The nascent binary was ejected from a dynamically unstable multiple protostellar system, leading to the loss of its accretion envelope before the secondary component could acquire enough mass to become a star.

2. Collision model. The binary was prematurely ejected from a larger protostellar system by the close passage of another star.

3. Photoevaporation model. The accretion envelope around the binary was photoevaporated by the nearby presence of a massive star in the birth cluster before the secondary could accrete enough mass to become a star.

4. "Star-like" model. The object formed as a normal stellar binary with low-mass components.

The embryo rejection model predicts (Bate et al. 2002) that the maximum separation of binaries surviving this process is $a_{\max } \sim 10-20 \mathrm{AU}$. We cannot rule out this scenario as we only measure the projected separation of the lens, which is nevertheless below $a_{\max }$. Since MOA-2010-BLG-073L is a field 
object, we have no information regarding the proximity of other stars during its birth, so the collisional and photoevaporation models are equally plausible. However, we note that Whitworth \& Stamatellos (2006) derived a minimum mass for primary fragmentation components of $0.004 M_{\odot} \sim 4.2 M_{\mathrm{J}}$. As this threshold is below the mass of MOA-2010-BLG-073L b, the simplest explanation is that the companion is an extremely low mass product of star formation. However, we note that the mass ratio of this system would match that of an object in the brown dwarf desert if the host were a more massive star. Recent surveys (e.g., Dieterich et al. 2012; Evans et al. 2012, though restricted to massive companions with $a>10 \mathrm{AU}$ ) hint that the brown dwarf desert may extend to M-dwarfs and beyond $3 \mathrm{AU}$, which would make MOA-2010-BLG-073L a rare example of its type.

R.A.S. is grateful to the Chungbuk University group and especially to C. Han and J.-Y. Choi for their advice and hospitality in Korea, where much of this work was completed. R.A.S. also expresses appreciation for Y. Tsapras, K. Horne, M. Hundertmark, S. Dong, and P. Fouqué for many useful discussions. K.A., D.M.B., M.D., K.H., M.H., C.L., C.S., R.A.S., and Y.T. thank the Qatar Foundation for support from QNRF grant NPRP-09-476-1-078. Work by C. Han was supported by the Creative Research Initiative Program (2009-0081561) of the National Research Foundation of Korea. C.S. received funding from the European Union Seventh Framework Programme (FP7/2007-2013) under grant agreement no. 268421. The OGLE project has received funding from the European Research Council under the European Community's Seventh Framework Programme (FP7/2007-2013)/ERC grant agreement no. 246678 to AU. This publication makes use of data products from the Exoplanet Encyclopeida and the Two Micron All Sky Survey, which is a joint project of the University of Massachusetts and the Infrared Processing and Analysis Center/California Institute of Technology, funded by the National Aeronautics and Space Administration and the National Science Foundation. M.O.A. acknowledges funding JSPS20340052, JSPS22403003, and JSPS19340058. T.C.H. gratefully acknowledges financial support from the Korea Research Council for Fundamental Science and Technology (KRCF) through the Young Research Scientist Fellowship Program. C.U.L. and T.C.H. acknowledge financial support from KASI (Korea Astronomy and Space Science Institute) grant number 2012-1-410-02. D.R. (boursier FRIA), F.F. (boursier ARC), and J. Surdej acknowledge support from the Communauté francaise de Belgique-Actions de recherche concertées-Académie universitaire Wallonie-Europe. A. Gould acknowledges support from NSF AST-1103471. B. S. Gaudi, A. Gould, and R. W. Pogge acknowledge support from NASA grant NNX12AB99G. Work by J. C. Yee is supported by a National Science Foundation Graduate Research Fellowship under grant No. 2009068160. S.D. is supported through a Ralph E. and Doris M. Hansmann Membership at the IAS and NSF grant AST-0807444.

Facilities: FTN, Liverpool:2m, CTIO:1.3m, MtCanopus:1m, Elizabeth, Danish 1.54m Telescope

\section{REFERENCES}

Albrow, M. D., Beaulieu, J.-P., Caldwell, J. A. R., et al. 1999, ApJ, 522, 1022 Albrow, M. D., Horne, K., Bramich, D. M., et al. 2009, MNRAS, 397, 2099 Alves, D. R., Rejkuba, M., Minniti, D., \& Cook, K. H. 2002, ApJL, 573, 51 Bachelet, E., Shin, I.-G., Han, C., et al. 2012, ApJ, 754, 73

Baraffe, I., Chabrier, G., \& Barman, T. 2010, RPPh, 73, 016901

Bate, M. R., Bonnell, I. A., \& Bromm, V. 2002, MNRAS, 332, L65
Batista, V., Gould, A., Dieters, S., et al. 2011, A\&A, 529, 102

Beaulieu, J.-P., Bennett, D. P., Fouqué, P., et al. 2006, Natur, 439, 437

Béjar, V. J. S., Zapatero Osorio, M. R., Rebolo, R., et al. 2012, ApJ, 743, 64

Bensby, T., Adén, D., Meléndez, J., et al. 2011, A\&A, 533, 134

Bessell, M. S., \& Brett, J. M. 1988, PASP, 100, 1134

Bond, I., Abe, F., Dodd, R. J., et al. 2001, MNRAS, 327, 868

Bonfils, X., Delfosse, X., Udry, S., et al. 2011, A\&A, submitted (arXiv:1111.5019)

Bonnell, I. A., Clark, P., \& Bate, M. R. 2008, MNRAS, 389, 1556

Boss, A. P. 2006, ApJ, 643, 501

Bramich, D. M. 2008, MNRAS, 386, L77

Burrows, A., Hubbard, J. I., Lunine, J. I., et al. 2001, RvMP, 73, 719

Chabrier, G., Baraffe, I., Allard, F., et al. 2005, in ASP Conf. Ser., Resolved Stellar Populations, ed. D. Valls-Gabaud \& M. Chavez (San Francisco, CA: ASP)

Chabrier, G., Leconte, J., \& Baraffe, I. 2011, in IAU Symp. 276, The Astrophysics of Planetary Systems: Formation, Structure, and Dynamical Evolution, ed. A. Sozzetti, M. G. Lattanzi, \& A. P. Boss (Cambridge: Cambridge Univ. Press), 171

Ciechanowska, A., Pietrzynski, G., Wyrzykowski, L., et al. 2006, AcA, 56,219

Cumming, A., Butler, R. P., Marcy, G. W., et al. 2008, PASP, 120, 531

Dieterich, S. B., Henry, T. J., Golimowski, D. A., et al. 2012, AJ, 144, 64

Dominik, M., Jørgensen, U. G., Rattenbury, N. J., et al. 2010, AN, 331, 671

Dong, S., DePoy, D. L., Gaudi, B. S., et al. 2006, ApJ, 642, 842

Dong, S., Gould, A., Udalski, A., et al. 2009, ApJ, 695, 970

Evans, T. M., Ireland, M. J., Kraus, A. L., et al. 2012, ApJ, 744, 120

Eyer, L., \& Blake, C. 2005, MNRAS, 358, 30

Fischer, D. A., \& Valenti, J. 2005, ApJ, 622, 1102

Forveille, T., Bonfils, X., Lo Curto, G., et al. 2011, A\&A, 526, A141

Gonzalez, O. A., Rejkuba, M., \& Zoccali, M. 2012, A\&A, 543, A13

Gould, A. 2008, ApJ, 681, 1598

Gould, A., Udalski, A., An, D., et al. 2006, ApJL, 644, 37

Heyrovský, D. 2007, ApJ, 656, 483

Howard, A. W., Marcy, G. W., Bryson, S. T., et al. 2012, ApJS, 201, 15

Ida, S., \& Lin, D. N. C. 2004, ApJ, 616, 567

Johnson, J. A., Aller, K. M., Howard, A. W., et al. 2010, PASP, 122, 905

Jorissen, A., Mowlavi, N., Sterken, C., \& Manifroid, J. 1997, A\&A, 324, 578

Kayser, R., Refsdal, S., \& Stabell, R. 1986, A\&A, 166, 36

Kervella, P., Bersier, D., Mourard, D., et al. 2004, A\&A, 428, 587

Kirkpatrick, J. D., Barman, T. S., Burgasser, A., et al. 2006, ApJ, 639, 1120

Kiss, L. L., Szabó, Gy. M., \& Bedding, T. R. 2006, MNRAS, 372, 1721

Klein, R., Apai, D., Pascucci, I., et al. 2003, ApJL, 593, 57

Kroupa, P., \& Bouvier, J. 2003, MNRAS, 346, 369

Kurucz, R. L. 1979, ApJS, 40, 1

Laughlin, G., Bodenheimer, P., \& Adams, F. C. 2004, ApJL, 612, 73

Luhman, K. L. 2012, ARA\&A, 50, 65

Maldonado, J., Eiroa, C., Villaver, E., et al. 2012, A\&A, 541, 40

Marcy, G. W., \& Butler, R. P. 2000, PASP, 112, 137

Marois, C., MacIntosh, B., \& Barman, T. 2008, Sci, 322, 1348

Miyake, N., Udalski, A., Sumi, T., et al. 2012, ApJ, 752, 82

Mordasini, C., Alibert, Y., \& Benz, W. 2009, A\&A, 501, 1139

Nataf, D. M., Gould, A., Fouqué, P., et al. 2012, ApJ, submitted (arXiv:1208.1263)

Park, B.-G., DePoy, D. L., Gaudi, B. S., et al. 2004, ApJ, 609, 166

Pejcha, O., \& Heyrovský, D. 2009, ApJ, 690, 1772

Penny, M. T., Mao, S., \& Kerins, E. 2010, MNRAS, 412, 607

Sahlmann, J., Ségransan, D., Queloz, D., et al. 2010, A\&A, 525, A95

Santos, N. C., Israelian, G., \& Mayor, M. 2001, A\&A, 373, 1019

Schneider, J., Dedieu, C., Le Sidaner, P., et al. 2011, A\&A, 532, A79

Scholz, A., Jayawardhana, R., \& Wood, K. 2006, ApJ, 645, 1498

Schwarzenberg-Czerny, A. 1996, ApJL, 460, 107

Shin, I.-G., Han, C., Choi, J.-Y., et al. 2012a, ApJ, 746, 127

Shin, I.-G., Han, C., Gould, A., et al. 2012b, ApJ, 760, 116

Skowron, J., Udalski, A., Gould, A., et al. 2011, ApJ, 738, 87

Skrutskie, M. F., Cutri, R. M., Stiening, R., et al. 2006, AJ, 131, 1163

Stanek, K. Z., \& Garnavich, P. M. 1998, ApJ, 503, 131

Sumi, T., Bond, I. A., \& Dodd, R. J. 2003, ApJ, 591, 204

Sumi, T., Kamiya, K., Bennett, D. P., et al. 2011, Natur, 473, 349

Tsapras, Y., Street, R., Horne, K., et al. 2009, AN, 330, 4

Udalski, A. 2003, AcA, 53, 291

Whitworth, A. P., \& Stamatellos, D. 2006, A\&A, 458, 817

Williams, J. P., \& Cieza, L. A. 2011, ARA\&A, 49, 67

Woźniak, P. R., Williams, S. J., Vestrand, W. T., \& Gupta, V. 2004, AJ, 128, 2965

Wray, J. J., Eyer, L., \& Paczyński, B. 2004, MNRAS, 349, 1059

Yee, J. C., Shvartzvald, Y., Gal-Yam, A., et al. 2012, ApJ, 755, 102

Zhou, J.-L., Xie, J-W., Liu, H-G., et al. 2012, RAA, 12, 1081 